zen ist eine offensichtliche Parallele zu der bei Licht und Dunkelheit ebenfalls auffälligen ${ }^{14} \mathrm{C}$-Aufnahme in verschiedene Verbindungen $(\mathrm{C}$ a lvin und Mitarbb.; C a lv in ${ }^{16,6}$ ).

Wurde die ${ }^{32} \mathrm{P}$-Aufnahme in Gegenwart von Glucose bei unseren Algen untersucht, so ergab sich der bemerkenswerte Befund, daß nunmehr keine zusätzliche lichtabhängige Phosphorylierung gemessen werden konnte. Es muß also sehr genau zwischen vorherigen Glucosegaben und mit der ${ }^{32} \mathrm{P}$-Aufnahme gleichzeitigen Glucosegaben unterschieden werden. Nun ist durch $\mathrm{K}$ a n d l e r ${ }^{11}$ festgestellt worden, daß bei Algen in schwachem Licht eine erhöhte Glucoseaufnahme in phosphathaltiger Nährlösung stattfindet. Diese Ergebnisse konnten wir durchaus bestätigen; bei hoher Lichtintensität ist die Glucoseaufnahme bei Ankistrodesmus sogar sehr erheblich gesteigert ${ }^{10}$. Auf Grund unserer mitgeteilten Befunde ist es jedoch nicht möglich, aus einer derart gesteigerten Glucoseaufnahme ohne weiteres auf eine zusätzliche Photosynthese-Phosphorylierung bzw. vermehrte lichtabhängige Phosphorylierung zu schließen, wie es $\mathrm{K}$ a n d l e r für möglich hält und zum Ausgangspunkt seiner weiteren Überlegungen macht. Da wir festgestellt haben, daß bei der Verarbeitung der vor der ${ }^{32} \mathrm{P}$-Aufnahme gegebenen Glucose eine meßbare Verstärkung der lichtabhängigen Phosphorylierung eintritt, diese aber dann fehlt, wenn die Glucose auch noch in die Zelle aufgenommen werden muß, liegt es nahe, diesen Effekt mit der Glucoseaufnahme als solcher in Beziehung zu setzen. Da die Glucoseaufnahme (z. B. nach R oth s te in ${ }^{12}$ ) einen an Zellgrenzschichten verlaufenden, Energie bedürftigen

16 M. Calvin, J. A. B a s s a m, A. A. B enson, V. H. Lynch, C. O u ellet, L. S chou, W. S tepka u. N. E. Tolbert. Symp. Soc. Exp. Biol. 5, 284 [1951]. und wohl sicher energiereiches Phosphat verbrauchenden Prozeß darstellt, fragt es sich, woher die zur vermehrten Glucoseaufnahme im Licht erforderliche Energie stammt. Sie könnte aus einem im Licht zusätzlich stattfindenden Abbau der bereits aufgenommenen Glucose oder aus erhöhter endogener Atmung, die nach P ir s o ${ }^{17}$ bei Glucosegaben weiterläuft, stammen, dann aber müßte solch einem Vorgang eine vermehrte Phosphorylierung entsprechen. Da diese aber nicht meßbar ist, bleibt nichts anderes übrig, als anzunehmen, daß entweder doch eine vermehrte Phosphorylierung vorhanden ist, die aber infolge von Grenzflächenvorgängen bei unserer Analyse, wie oben schon erwähnt, nicht in der Zelle selbst gemessen werden kann - auf die Möglichkeit solcher Prozesse weist u. a. R oth ste in ${ }^{12}$ hin oder die Phosphorylierungs-Energie wird doch der Photosynthese entnommen. Das würde aber bedeuten, daß dann die Phosphorylierungs-Energie nicht mehr der $\mathrm{CO}_{2}$-Assimilation zur Verfügung steht. Es wäre also $\mathrm{zu}$ erwarten, daß die $\mathrm{CO}_{2}$-Assimilation durch Glucoseaufnahme im Licht gehemmt werden kann. Dieses würde bedeuten, daß die bei der Photosynthese gewonnene Energie zu anderen Aufgaben als nur zur $\mathrm{CO}_{2}$-Assimilation verwendet werden kann, eine schon verschiedentlich geäußerte Vermutung (vgl. z. B. K a n d le ${ }^{8}$ ). In der folgenden Mitteilung werden wir zeigen, daß die $\mathrm{CO}_{2}$-Abgabe bei Belichtung unserer Ankistrodesmus-Kulturen und bei gleichzeitiger Glucosegabe in der Tat sehr stark gehemmt werden kann.

Die vorliegenden Untersuchungen wurden durch eine wertvolle Beihilfe der Deutschen Forschungs gemeinschaft, der wir hierfür sehr zu Dank verpflichtet sind, ermöglicht.

17 A. Pirson, A. L. Daniel u. E. W. B e cker. Arch. Mikrobiol. 22, 214 [1955].

\title{
Zur Chemie von Polyhalocyclopentadienen
}

\section{Mitt. XII: Tetrahalocyclopentadiene}

\author{
Von R. Riemschneider, G. Engelmann und K. Rosswitz \\ Aus dem Institut für Organische Chemie der Freien Universität Berlin-Dahlem \\ (Z. Naturforschg. 11 b, 172-174 [1956]; eingegangen am 11. Februar 1955)
}

Herstellung und Reaktionen einiger 1.2.3.4-Tetrahalo-cyclopentadiene-(1.3) werden beschrieben.

$\mathrm{H}_{\mathrm{p}}^{\mathrm{e}}$ exabromcyclopentadien (I) und Hexachlorcyclopentadien (II) verhalten sich gegenüber Reduktionsmitteln nicht völlig analog. Dies zeigte sich, als wir versuchten, das Br-Analoge des Pentachlorcyclo- pentadiens ${ }^{1}$ (III) aus I herzustellen. 2-stdg. Einwirkung von nasz. Wasserstoff aus $\mathrm{Zn}$ und $\mathrm{HCl}$ auf $\mathrm{I}$ in Petroläther bei $40^{\circ}$ führte nur zu einem der drei aus 1 Mitt. V, Z. Naturforschg. 6 b, 463 [1951]. 
II erhaltenen Reaktionsprodukte, und zwar zum Tetrabromcyclopentadien (IV $=\mathrm{C}_{5} \mathrm{H}_{2} \mathrm{Br}_{4}$ ). Aus II hatten wir unter bestimmten Bedingungen nicht nur $\mathrm{C}_{5} \mathrm{H}_{2} \mathrm{Cl}_{4}(\mathrm{~V})$, sondern auch III und $\mathrm{C}_{10} \mathrm{H}_{2} \mathrm{Cl}_{8}$ erhalten, von denen III ebenfalls $\mathrm{zu} \mathrm{V}$ reduziert werden konnte. Dieser Versuch stützt die bereits früher ${ }^{1}$ für III angenommene 1.2.3.4.5-Stellung der Cl-Atome. Gegenüber $\mathrm{Zn}$ in siedendem Methanol verhielten sich die genannten fünf Polyhalocyclopentadiene insofern ancilog, als in jedem Falle bis zu 15\% Cyclopentadien aus dem Reaktionsprodukt isoliert werden konnte, das durch Umsetzung mit II zu $\mathrm{C}_{10} \mathrm{H}_{6} \mathrm{Cl}_{6}{ }^{2}$ charakterisiert wurde.

$\mathrm{C}_{5} \mathrm{H}_{2} \mathrm{Br}_{4}$ (IV) haben wir auf Grund seines Verhaltens gegenüber Halogenen, Aldehyden und ungesättigten Verbindungen als 1.2.3.4-Tetrabrom-cyclopentadien-(1.3) identifizieren können: IV addiert Chlor unter Bildung von Tetrabromdichlorcyclopenten (VI). Entsprechende Umsetzungen von Chlor bzw. Brom und $\mathrm{V}$ führten zum $\mathrm{C}_{5} \mathrm{H}_{2} \mathrm{Cl}_{6}$ (VII) bzw. $\mathrm{C}_{5} \mathrm{H}_{2} \mathrm{Cl}_{4} \mathrm{Br}_{2}$ (VIII), während bei der Behandlung von IV, V, III und I mit flüssigem Chlor im Einschlußrohr in jedem Falle Oktachlorcyclopenten (IX) entstand. Mit Chlorbenzaldehyden vereinigt sich IV unter Wasserabspaltung zu den erwarteten Fulvenen (X). Aus den bei der Umsetzung von IV bzw. V mit Cyclopentadien und Alkylcyclopentadienen resultierenden Reaktionsprodukten ließen sich die Addukte $\mathrm{C}_{10} \mathrm{H}_{7} \mathrm{RX}_{4}$ $\left(\mathrm{X}=\mathrm{Cl}, \mathrm{Br} ; \mathrm{R}=\mathrm{H}, \mathrm{C}_{2} \mathrm{H}_{5}, \mathrm{C}_{3} \mathrm{H}_{7}, \mathrm{C}_{4} \mathrm{H}_{9}\right.$ u. a.) abtrennen. Zur Identifizierung wurde das Addukt $\mathrm{C}_{10} \mathrm{H}_{8} \mathrm{Cl}_{4}$ mit II im Rohr auf $150^{\circ}$ erhitzt, wobei - in Analogie zur Umsetzung von II mit $\mathrm{C}_{10} \mathrm{H}_{6} \mathrm{Cl}_{6}{ }^{2}-$ das Addukt $\mathrm{C}_{15} \mathrm{H}_{8} \mathrm{Cl}_{10}$ entstand. Mit der Prüfung der Frage, ob $\mathrm{V}$ gegenüber Cyclopentadien und Substitutionsprodukten als Dien oder Philodien fungiert, sind wir noch beschäftigt. Bei der Umsetzung von $\mathrm{V}$ mit ausgesprochenen Philodienen ist $\mathrm{V}$ das Dien; z. B. entsteht aus $\mathrm{V}$ und $p$-Benzochinon das Addukt $\mathrm{C}_{11} \mathrm{H}_{6} \mathrm{O}_{2} \mathrm{Cl}_{4}(\mathrm{XV})$.

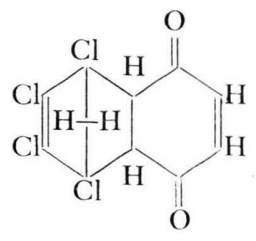

$\mathrm{XV}$

$\because$ Mitt. VI, Mh. Chem. 83, 802 [1952]. Vgl. auch Z. Naturforschg. 6 b, 395 [1951] u. Mitt. Physiolog.-Chem. Inst. Bln. R 11, Okt. 1947, C. A. 1955, 8216; Mh. Chem. 86, 879 [1955].
Da V - im Gegensatz zu II - beim Erhitzen unter teilweiser Zersetzung mit sich selbst reagiert (Dimeres vom Schmp. $193^{\circ}$ ), addiert es sich nicht so allgemein an Doppelbindungen wie II ${ }^{2}$.

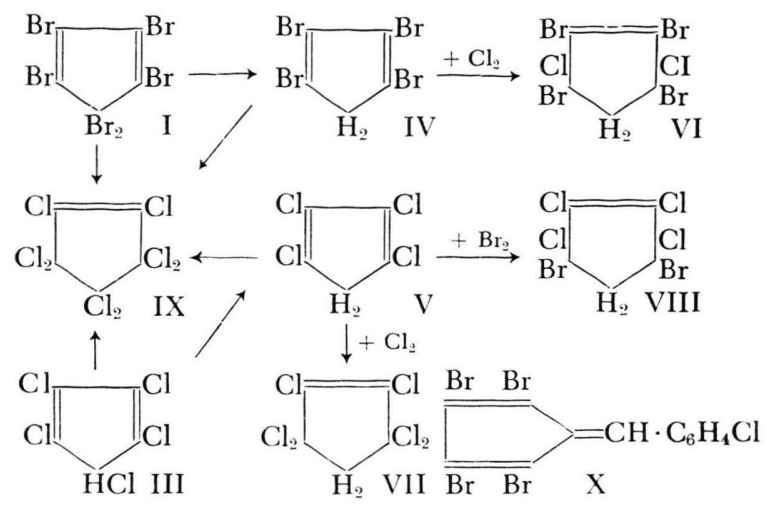

\section{Beschreibung der Versuche}

1.2.3.4-Tetrabrom-cyclopen ta dien-(1.3) (IV) a us Hexabromcyclopentadien (I)

Eine Lösung von 5 g I vom Schmp. $88-89^{\circ} 3$ in $40 \mathrm{ml}$ Petroläther $\left(40-60^{\circ}\right)$ wird mit $5 \mathrm{~g}$ Zinkstaub (aktiviert) versetzt. Dann werden unter kräftigern Rühren bei $40^{\circ}$ im Laufe von 2 bis 3 Stdn. $20 \mathrm{ml}$ 25-proz. $\mathrm{HCl}$ hinzugetropft. Nach Abfiltrieren und Auswaschen des nicht umgesetzten Zinkstaubs mit Petroläther wird die Petrolätherschicht abgetrennt, gewaschen, mit entwässertem Natriumsulfat getrocknet und stark eingeengt. Die bei Eiskühlung ausfallenden weißen Kristalle werden zur Entfernung des evtl. noch vorhandenen I in heißem $\mathrm{CHCl}_{3}$ gelöst. Beim Abkühlen fällt nur IV aus. Nach mehrmaligem Umkristal-

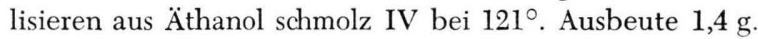

$$
\begin{array}{lllllll}
\mathrm{IV}=\mathrm{C}_{5} \mathrm{H}_{2} \mathrm{Br}_{4}(382) & \text { Ber. } & \text { C } 15,7 & \mathrm{H} & 0,5 & \mathrm{Br} & 83,7 . \\
& \text { Gef. } & \text { C } 15,6 & \text { H } & 0,5 & \text { Br } 83,8 .
\end{array}
$$

Gegenüber Alkalien ist IV sehr empfindlich: Mit 0,1-n. alkahol. KOH bildeten sich blaugrüne Lösungen, die rasch dunkler werden. Auf Grund dieses Verhaltens von IV ist verständlich, daß IV nicht durch Einwirkung von alkalischer $\mathrm{OBr}^{\prime}$-Lösung auf Cyclopentadien hergestellt werden kann ${ }^{4}$.

1.2.3.4-Tetrachlor-cyclopentadien-(1.3) (V) haben wir in entsprechender Weise aus Hexachlorcyclopentadien (II) hergestellt: Reaktionstemperatur $20^{\circ}$, -zeit 1,5 Stdn. bei einem Ansatz von 16,3 g II vom Sdp. $100-104^{\circ}$ bei $11 \mathrm{~mm}^{3}, 30 \mathrm{ml}$ Petroläther, $10 \mathrm{~g} \mathrm{Zn}$ und $25 \mathrm{ml} \mathrm{HCl}$. V vom Schmp. $63-64^{\circ}$ wurde in ein : Ausbeute von $53 \%$ erhalten.

3 Hergestellt nach der in Mitt. VII tieser Reihe veröffentlichten Vorschrift: Chim. e Ind. : :ilano] 34, 266 [1952]. In Versuch $15 \mathrm{mu}$ es dort $77 \mathrm{ml}$ statt $77 \mathrm{~g}$ heißen.

4 Vgl. 1. c. ${ }^{3}$. 
1.2.3.4- Tetrachlor-cyclopentadien-

(1.3) (V) a u s 1.2.3.4.5-Pentachlorcyclopentadien - (1.3) (I I I)

$24 \mathrm{~g}$ III vom Sdp. $90-94^{\circ}$ bei $11 \mathrm{~mm}^{3}$ in $75 \mathrm{ml}$ Petroläther $\left(40-60^{\circ}\right)$ werden mit $20 \mathrm{~g}$ Zinkstaub versetzt und unter kräftigem Rühren bei $50^{\circ}$ im Verlauf von $2,5 \mathrm{Stdn}$. $70 \mathrm{ml}$ 25-proz. $\mathrm{HCl}$ hinzugetropft. Während der Umsetzung wird die Lösung rötlich. Nach Abfiltrieren und Auswaschen des nicht umgesetzten $\mathrm{Zn}$ mit Petroläther wird die abgetrennte und gewaschene Petrolätherlösung nach Trocknen auf 15-20 ml eingeengt. Beim Abkühlen in EisKochsalz scheiden sich V-Kristalle aus, die nach mehrmaligem Umkristallisieren aus Petroläther bei 63-64 schmolzen. Ausbeute: $14 \mathrm{~g}$.

$$
\begin{array}{llllllll}
\mathrm{V}-\mathrm{C}_{3} \mathrm{H}_{2} \mathrm{Cl}_{4}(204) & \text { Ber. } & \text { C } 29,5 & \mathrm{H} & 1,0 & \mathrm{Cl} & 69,6 . \\
& \text { Gef. } & \text { C } 29,6 & \mathrm{H} & 0,9 & \mathrm{Cl} & 69,8 .
\end{array}
$$

Halogenierungen von Tetrahalocyclopentadienen

a) Addition von Brom an V: Zu einer Lösung von $3 \mathrm{~g}$ $\mathrm{V}$ in $20 \mathrm{ml}$ Eisessig werden unter Schütteln $3 \mathrm{~g}$ Brom gegeben, wobei unter starker Wärmeentwicklung sofortige Entfärbung eintritt. Die in der Kälte ausfallenden glänzenden Kristalle schmelzen nach Umkristallisieren aus Äthanol bei $70^{\circ}$. Ausbeute: $4,3 \mathrm{~g}$ VIII.

$$
\begin{aligned}
& \text { VIII }=\mathrm{C}_{5} \mathrm{H}_{2} \mathrm{Cl}_{4} \mathrm{Br}_{2}(364) \\
& \text { Ber. C 16,5 H 0,55 Cl 39,0 Br 43,9. } \\
& \text { Gef. C 16,8 H } 0,65 \text { Cl 39,1 } \mathrm{Br} 44,1 \text {. }
\end{aligned}
$$

b) Addition von Chlor an V: In eine Lösung von $4 \mathrm{~g} \mathrm{~V}$ in $40 \mathrm{ml} \mathrm{CCl}$ sird bei Zimmertemperatur im Verlauf von 1 Stde. ein langsamer Chlorstrom geleitet. Der nach Absaugen des Lösungsmittels hinterbleibende Rückstand wird mehrmals aus Äthanol umkristallisiert: $3,4 \mathrm{~g}$ VII vom Schmp. $41^{\circ}$.

$$
\begin{array}{llllllll}
\text { VII }-\mathrm{C}_{5} \mathrm{H}_{2} \mathrm{Cl}_{6}(275) & \text { Ber. } & \text { C } 21,9 & \mathrm{H} & 0,7 & \mathrm{Cl} & 77,5 . \\
& \text { Gef. } & \text { C } 21,7 & \mathrm{H} & 0,8 & \mathrm{Cl} & 77,8 .
\end{array}
$$

c) Addition von Chlor an IV: Ausführung wie unter b) angegeben

$$
\begin{aligned}
& \mathrm{VI}-\mathrm{C}_{5} \mathrm{H}_{2} \mathrm{Cl}_{2} \mathrm{Br}_{4} \text { (453) } \\
& \text { Ber. C 13,2 H } 0,4 \quad \mathrm{Cl} \quad 15,3 \text { Br } 70,6 \text {. } \\
& \text { Gef. C } 13,0 \text { H } 0,5 \text { Cl } 15,7 \text { Br } 70,1 \text {. }
\end{aligned}
$$

d) Oktachlorcyclopenten (IX) aus IV: $7 \mathrm{~g}$ IV und $7 \mathrm{ml}$ flüssiges Chlor werden im Einschlußrohr zehn Tage im Sonnenlicht aufbewahrt. Das ölige Reaktionsprodukt (intensiv rotbraun gefärbt) kristallisiert beim Anreiben mit wenig $\mathrm{CHCl}_{3}$ und wird aus Äthanol umkristallisiert: $4,1 \mathrm{~g}$ IX vom Schmp. $42^{\circ}$.

$$
\begin{array}{llll}
\mathrm{IX}=\mathrm{C}_{5} \mathrm{Cl}_{8}(344) & \text { Ber. } & \text { C } 17,5 & \mathrm{Cl} 82,5 . \\
& \text { Gef. } & \text { C } 17,4 & \mathrm{Cl} 82,8 .
\end{array}
$$

Brom konnte qualitativ nicht mehr im Reaktionsprodukt IX nachgewiesen werden. Bei der Behandlung von $5 \mathrm{~g} \mathrm{I}$, $\mathrm{V}$ oder III mit $5 \mathrm{ml}$ flüssigem Chlor unter entsprechenden
Bedingungen entstand ebenfalls IX. Ausbeute zwischen 3 und $3,6 \mathrm{~g}$.

Umsetzung von 1.2.3.4-Tetrabromcyclopentadien-(1.3) (IV) mit Cyclopentadien 5

$\mathrm{Zu} 38 \mathrm{~g}$ IV in $15 \mathrm{ml}$ Toluol werden in der Hitze im Verlauf von 4 Stdn. $7 \mathrm{~g}$ frisch hergestelltes Cyclopentadien getropft und noch 5 Stdn. gelinde erhitzt. Der nach Abdampfen des Lösungsmittels erhaltene Rückstand wird unter Zusatz von Tierkohle mehrmals mit je $50 \mathrm{ml}$ Methanol erhitzt und das Addukt aus Methanol umkristallisiert. Wir erhielten $9 \mathrm{~g} \mathrm{C}_{10} \mathrm{H}_{8} \mathrm{Br}_{4}$ schmelzend ab $115^{\circ}(\mathrm{XI})$.

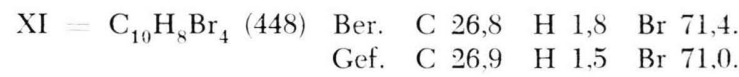

Umsetzung von 1.2.3.4-Tetrachlor-cyclopentadien-(1.3) (V) mit Cyclopentadien und Alkylcyclopentadienens

$\mathrm{Zu} 20 \mathrm{~g} \mathrm{~V}$ in $10 \mathrm{ml}$ Toluol werden in der Hitze im Verlauf von 4 Stdn. $7 \mathrm{~g}$ frisch hergestelltes Cyclopentadien (bzw. subst. Cyclopentadien in entsprechender Menge) hinzugetropft und wie üblich aufgearbeitet. Das redestillierte Addukt $\mathrm{C}_{10} \mathrm{H}_{8} \mathrm{Cl}_{4}$ (XII) ging bei $1 \mathrm{~mm}$ zwi schen 95 und $97^{\circ}$ über. Ausbeute: $22 \mathrm{~g}$.

$$
\text { XII }=\mathrm{C}_{10} \mathrm{H}_{8} \mathrm{Cl}_{4}(270) \begin{array}{llllll}
\text { Ber. } & \text { C } 44,5 & \text { H } 3,0 & \text { Cl } 52,5 . \\
& \text { Gef. } & \text { C } 44,3 & \text { H } 3,1 & \text { Cl } 52,7 .
\end{array}
$$

Das aus V und Äthylcyclopentadien erhaltene Addukt $\mathrm{C}_{12} \mathrm{H}_{12} \mathrm{Cl}_{4}$ siedete bei $0,6 \mathrm{~mm}$ zwischen 110 und 113 (XIII). Das homologe $\mathrm{C}_{13} \mathrm{H}_{14} \mathrm{Cl}_{4}$ (XIV) ging bei $0,8 \mathrm{~mm}$ zwischen 123 und $127^{\circ}$ über.

$\begin{array}{lllllll}\text { XIII }=\mathrm{C}_{12} \mathrm{H}_{12} \mathrm{Cl}_{4}(298) & \text { Ber. } & \text { C } 48,4 & \mathrm{H} & 4,1 & \mathrm{Cl} & 47,6 . \\ & \text { Gef. } & \text { C } 48,0 & \mathrm{H} & 3,9 & \mathrm{Cl} & 47,8 . \\ & & & & & & \\ \text { XIV }=\mathrm{C}_{13} \mathrm{H}_{14} \mathrm{Cl}_{4}(312) & \text { Ber. } & \text { C } 50,0 & \mathrm{H} & 4,5 & \mathrm{Cl} & 45,4 .\end{array}$

Umsetzung von 1.2.3.4-Tetrachlor-cyclopentadien-(1.3) (V) mit $p$ - Chinon

$5 \mathrm{~g} \mathrm{~V}$ und 2,5 g $p$-Chinon werden in $10 \mathrm{ml}$ Toluol einige Stdn. zum Sieden erhitzt. Nachdem nicht umgesetztes $p$ Chinon durch Wasserdampfdestillation entfernt ist, wird ausgeäthert und das Addukt aus Äthanol umkristallisiert. Das gelbe Addukt $\mathrm{C}_{11} \mathrm{H}_{6} \mathrm{O}_{2} \mathrm{Cl}_{4}$ schmolz bei $171^{\circ}$ (XV). Ausbeute: $3,9 \mathrm{~g}$.

$$
\begin{aligned}
& \mathrm{XV}=\mathrm{C}_{11} \mathrm{H}_{6} \mathrm{O}_{2} \mathrm{Cl}_{4}(312) \quad \text { Ber. } \mathrm{C} 42,3 \quad \mathrm{H} 1,9 \text { Cl 45,5. } \\
& \text { Gef. C 42,5 H 2,0 } \mathrm{Cl} 45, \mathrm{~S} \text {. }
\end{aligned}
$$

Die vorstehend beschriebenen Versuche sind in den Jahren 1951 und 1952 ausgeführt worden. Wir danken der Deutschen Forschungsgemeinschaft für die finanzielle Förderung unserer Untersuchungen.

5 Analog der Umsetzung von Hexahalocyclopentadienen mit Cyclopentadien, l. c. ‥ 